\title{
Cerebral Abnormalities in Adults with Ataxia-Telangiectasia
}

\author{
D.D.M. Lin, P.B. Barker, H.M. Lederman, and T.O. Crawford
}

\begin{abstract}
SUMMARY: Ataxia-telangiectasia, an autosomal recessive disorder caused by defect of the ataxia-telangiectasia mutated gene, is characterized by progressive neurologic impairment with cerebellar atrophy, ocular and cutaneous telangiectasia, immunodeficiency, heightened sensitivity to ionizing radiation and susceptibility to developing lymphoreticular malignancy. Supratentorial brain abnormalities have been reported only rarely. In this study, brain MRI was performed in 10 adults with ataxia-telangiectasia having stable neurologic impairment. Intracerebral telangiectasia with multiple punctate hemosiderin deposits were identified in $60 \%$ of subjects. These lesions were apparently asymptomatic. They are similar in appearance to radiation-induced telangiectasia and to cryptogenic vascular malformations. Also noted, in the 2 oldest subjects, was extensive white matter T2 hyperintensity, and in 1 of these a space-occupying fluid collection consistent with transudative capillary leak and edema as evidenced by reduced levels of metabolites on MR spectroscopic imaging. Asymptomatic supratentorial vascular abnormalities appear to be common in adults with ataxia-telangiectasia.
\end{abstract}

ABBREVIATIONS: A-T = ataxia-telangiectasia; AFP = alpha-fetoprotein; $A T M=$ ataxia telangiectasia mutated; MRSI = MR spectroscopic imaging

A taxia-telangiectasia (A-T) is an autosomal recessive neurodegenerative disorder associated with a single defective gene localized to chromosome $11(11 \mathrm{q} 22-23)^{1}$ that is estimated to affect 1 in 40,000-300,000 people. ${ }^{2,3}$ The causative gene, termed ataxia telangiectasia mutated (ATM), is constitutively expressed in all eukaryotic cells and encodes a serine-threonine kinase key to a number of important cellular responses, including the DNA damage response and associated cell-cycle checkpoint regulation. ${ }^{4}$ The range of clinical features manifested by people with A-T is similarly broad and dramatic, including telangiectatic vessels on the bulbar conjunctivae and skin, humeral and cell-mediated immunodeficiency, and an array of cerebellar and other neurologic

Received May 31, 2011; accepted after revision March 13, 2013.

From the Departments of Radiology and Radiological Science (D.D.M.L., P.B.B.), Pediatrics (H.M.L., T.O.C.), and Neurology (T.O.C.), Johns Hopkins University School of Medicine, Baltimore, Maryland.

This publication was made possible by the Johns Hopkins Institute for Clinical and Translational Research (ICTR), which is funded in part by grant number UL1 TR 000424-06 from the National Center for Advancing Translational Sciences (NCATS), a component of the National Institutes of Health (NIH), and the NIH Roadmap for Medical Research. Its contents are solely the responsibility of the authors and do not necessarily represent the official view of the Johns Hopkins ICTR, NCATS, or NIH. Additional support was provided by NIH grant P41EB015909 and the Ataxia Telangiectasia Children's Project, Boca Raton, Florida.

Please address correspondence to Doris D.M. Lin, MD, PhD, Russell H. Morgan Department of Radiology and Radiological Science, Johns Hopkins University School of Medicine, 600 North Wolfe St, Phipps B-100, Baltimore, MD 21287; e-mail: ddmlin@jhmi.edu

- Indicates open access to non-subscribers at www.ajnr.org

http://dx.doi.org/10.3174/ajnr.A3646 impairments. ${ }^{5}$ Affected persons also manifest both heightened sensitivity to ionizing radiation and enhanced susceptibility to malignant disease, chiefly lymphoreticular in nature. ${ }^{6-8}$

MR imaging is the favored technique of neuroimaging for patients suspected to have A-T because of its superior tissue contrast, as well as the absence of ionizing radiation. Cerebellar atrophy is the most consistent finding, though it is usually not present in the preschool years when most patients first seek neurologic consultation. ${ }^{9}$ The supratentorial brain is typically normal on MR imaging. ${ }^{10,11} \mathrm{~A}$ few cases have been reported of patients with A-T, mostly adults surviving into their second and third decades of life, in whom cerebral white matter abnormalities of 2 types have been described. One pattern consists of multiple $\mathrm{T} 1$ and $\mathrm{T} 2$ hypointense foci suggestive of hemosiderin, thought to be related to thrombosis and vascular leaks from multiple capillary telangiectasias. ${ }^{12}$ The other pattern, described in a single child and in a few adult patients, ${ }^{11,13-15}$ is T2/ FLAIR hyperintensity on long TR sequences that simulates leukodystrophy. The purpose of this study was to analyze the MR imaging features of the supratentorial brain in a small series of young adults with A-T, all otherwise healthy and neurologically stable for years, though they were manifesting substantial persistent neurologic deficits.

\section{Case Series}

This study was approved by the local institutional review board (NA_00014314 and NA_00012201). Ten adult participants with A-T (4 men, 6 women; mean age, $23.4 \pm 4.5$ years; age range, 


\begin{tabular}{|c|c|c|c|c|c|c|c|}
\hline Participant & Age/Sex & Age of Onset ${ }^{a}$ & $\mathrm{AFP}^{\mathrm{b}}(\mathrm{ng} / \mathrm{mL})$ & Chromatid Breaks/Cell' & SWI Lesions & WM T2 Hyperintensity & $\begin{array}{l}\text { Additional MRI } \\
\text { Findings }\end{array}$ \\
\hline 1 & $21 / \mathrm{F}$ & 1 & 498 & 2.02 & Multiple & None & - \\
\hline 2 & $21 / F$ & 1 & 123 & NA & 1 & None & - \\
\hline 3 & $25 / F$ & 2 & 1 & 2.20 & None & None & - \\
\hline 4 & $20 / M$ & 1 & 400 & NA & Multiple & None & - \\
\hline 5 & $22 / \mathrm{M}$ & 3 & 240 & NA & Multiple & None & - \\
\hline 6 & $23 / F$ & 1.5 & 224 & 1.48 & Several & None & - \\
\hline 7 & $19 / \mathrm{F}$ & 2 & 14 & 1.34 & None & None & - \\
\hline 8 & $21 / F$ & 1.5 & 384 & 2.58 & None & None & - \\
\hline 9 & $34 / M$ & 3 & 484 & 2.14 & Innumerable & Present & - \\
\hline 10 & $28 / M$ & 2 & 715 & 1.92 & Innumerable & Present & Cystic mass \\
\hline
\end{tabular}

Note:-NA indicates not available.

${ }^{\text {a }}$ Age (years) at first inquiry to physician for neurologic concern.

${ }^{b}$ Normal $<10 \mathrm{ng} / \mathrm{mL}$.

${ }^{c}$ Measured after in vitro exposure to x-irradiation (1.0 Gy); normal range, 0.06-0.34 (Thomas GH and Lederman HM, personal communication).

19-34 years; Table) constitute the case series here reviewed. Eight of these patients were enrolled in a research study ${ }^{16}$ to collect CSF, and they underwent MR imaging scans as a safety measure before lumbar puncture. The ninth participant was neurologically stable but underwent clinical MR imaging to evaluate chronic headaches. The final participant underwent MR imaging for clinical concerns but had experienced no new symptoms or signs in several years. The research brain MR imaging studies were performed at $3 \mathrm{~T}$ and consisted of T1, T2, and SWI. For the 2 clinical studies, in addition to conventional sequences, proton MR spectroscopic imaging (MRSI; TR, $1500 \mathrm{~ms}$; TE, $280 \mathrm{~ms}$ ) was acquired at $1.5 \mathrm{~T}$.

All participants were followed in the Ataxia-Telangiectasia Clinical Center at our institution and underwent MR imaging between 2005 and 2008. At the time of MR imaging evaluation, all participants had stable neurologic impairments of variable severity, including ataxia, dystonia, various forms of tremor, dysarthria, oculomotor abnormalities, distal diminished sensibility to light touch and position, and decreased or absent distal tendon reflexes. All used a wheelchair for mobility. The diagnosis of A-T was by fulfillment of established clinical criteria, ${ }^{5,9}$ including these and other characteristic clinical features, and laboratory studies of elevated alpha-fetoprotein (AFP); increased lymphocyte chromosomal breaks after in vitro radiation exposure; and, in some cases, demonstration of absent ATM protein on Western blot analysis or demonstration of predicted protein-null homozygous mutations of ATM (Table).

On MR imaging, all participants had manifest cerebellar atrophy, whereas the supratentorial brain showed no sign of volume loss. In 6 participants, SWI showed few to innumerable punctate signal voids, suggesting hemosiderin deposits, scattered throughout the cerebral white matter. Four of these 6 were in the research-acquired group, and in these 4 participants, the punctate lesions were inconspicuous on other pulse sequences, including T2-weighted FSE images. In the 2 participants (participants 9 and 10) scanned for clinical concerns (but, like the research-acquired group, absent of any recent change in neurologic function) additional findings of white matter T2 hyperintensity were present, surrounding and highlighting some of these lesions. No signal abnormalities were evident in the cerebellum or brain stem.

In participant 9 (Fig 1), a 34-year-old man who was evalu- ated for chronic headache, innumerable punctate lesions were best seen on SWI. Several clusters of these lesions were surrounded by T2/FLAIR hyperintensity in the white matter and were associated with mild mass effect (Fig 1A). In these regions of white matter hyperintense T2 signal abnormality-in contrast to the surrounding normal-appearing white matter- ${ }^{1} \mathrm{H}$ MRSI showed a diminution of all examined metabolites, suggesting reduced cellularity with edema, or perhaps gliosis (Fig $1 B)$. No increase of choline was evident, arguing against an active demyelinating process. No abnormal elevation of lactate was found in any of the brain regions or in the CSF.

In participant 10 (Fig 2), a 28-year-old man who underwent MR imaging simply because of concern for his advancing age, there was an ovoid T2 hyperintense and T1 hypointense spaceoccupying lesion lined by hemosiderin deposits and surrounded by vasogenic edema (Fig $2 A$ ). Some of the T2 dark lesions showed contrast enhancement, suggesting telangiectatic vessels. Within the surrounding white matter T2 hyperintensity, MRSI (Fig 2B) again showed the decrease of all metabolites in a pattern similar to that apparent in participant 9. The right frontal lesion was virtually devoid of any metabolites, consistent with a fluid collection.

\section{DISCUSSION}

This case series identifies a spectrum of supratentorial white matter findings in adults with A-T. Combined with the experience of a few reported individual cases, ${ }^{12,17}$ it is apparent that patients with A-T frequently manifest scattered small white matter hypointensities that most likely represent tiny hemosiderin deposits related to telangiectatic vessels. These lesions were most conspicuous on SWI. The presence of these asymptomatic lesions in 6 of 10 neurologically stable patients $>19$ years old contrasts with their absence in a younger reported cohort $^{18}$ of 8 patients with A-T (mean age, 13 years; age range, 9-19 years), as well as our general experience at the Johns Hopkins Ataxia Telangiectasia Clinical Center reviewing several hundred initial diagnostic MR imaging scans. Although age-related differences in sensitivity or other technical differences are possible confounders, the most plausible explanation is that these are acquired lesions, passing over a threshold of MR imaging detectability in early adult years.

Disabling mutation of the ATM gene responsible for A-T 

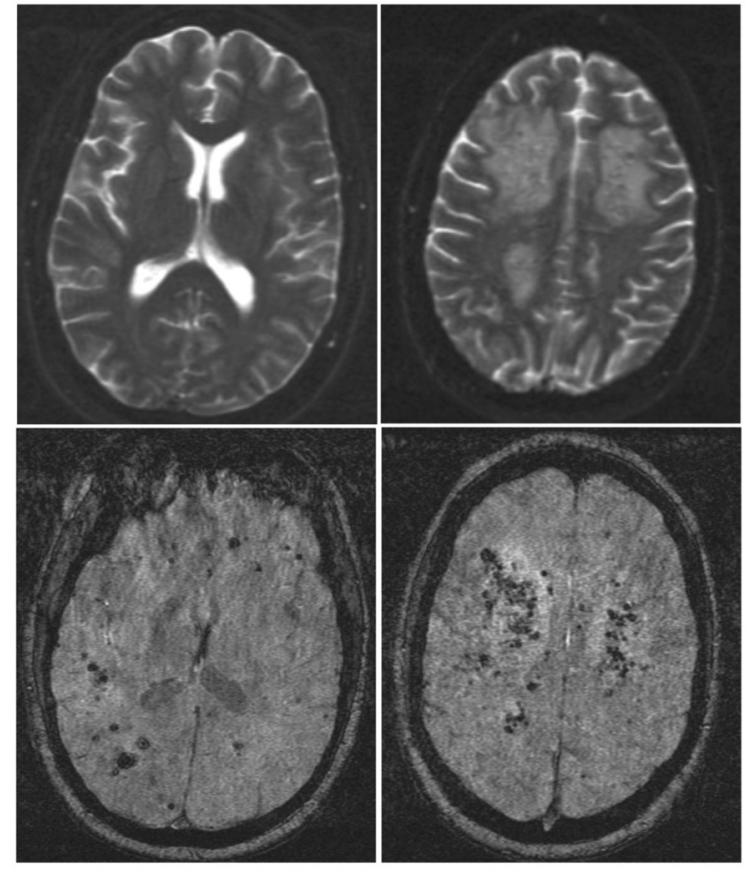

$\mathrm{B}$
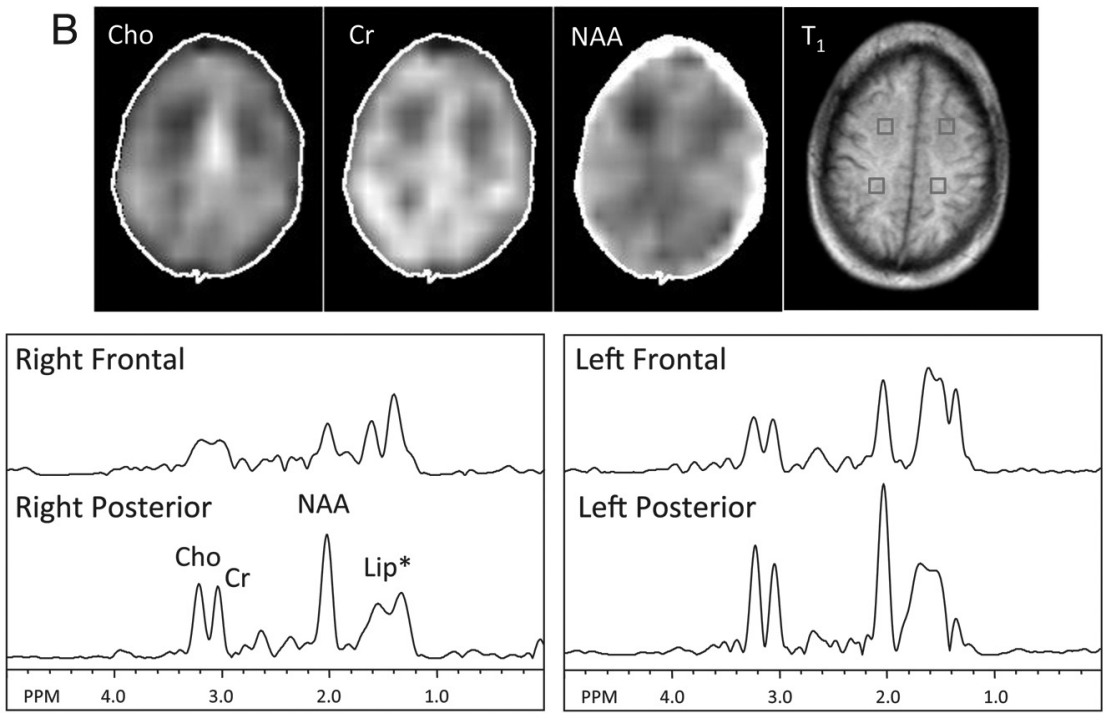

FIG 1. MR imaging and MRSI in participant 9, a 34-year-old man with A-T who had experienced chronic headaches. A, Top: T2-weighted images show multifocal confluent hyperintensity in the frontal and parietal white matter bilaterally. A few punctate hypointense foci suggestive of hemosiderin deposits can be identified in the left frontal white matter. Bottom: SWI shows innumerable foci of susceptibility consistent with hemosiderin deposits scattered throughout the bilateral cerebral white matter, some clustered within the confluent T2 hyperintensity. $B$, MRSI metabolite maps ( $C h o=$ choline, $\mathrm{Cr}=$ creatine, NAA $=\mathrm{N}$-acetylaspartate) and corresponding T1-weighted MR imaging showing voxel locations for selected spectra in the left and right frontal and posterior white matter. Lower levels of Cho, $\mathrm{Cr}$, and NAA are seen, particularly in the right frontal region corresponding to T2 hyperintense white matter, compared with the spectra obtained from the posterior centrum semiovale where more normal-appearing white matter was found. Note the broad peak (approximately 1.5 ppm) upfield from NAA in all brain regions is related to scalp lipid contamination that results from head motion during the scan.

has pleotrophic cellular effects. ${ }^{4,19,20}$ Among the most prominent of these is disruption of the cell-cycle arrest and other cellular responses to DNA damage. Identification of the specific impaired ATM-kinase pathway responsible for formation of deep white matter telangiectasia is unknowable, though this is an appropriate target for research. The ocular and cutaneous telangiectasias that are part of the defining feature of A-T appear in light-exposed superficial regions. A similar vascular pathologic pattern in ATM-deficient mice ${ }^{21}$ that arises in the retina with diminished retinal vasculature attenuation is asso- ciated with increased vascular endothelial growth factor expression (implicated in angiogenesis), decreased tight junction protein occludin expression, and perturbed astrocytic interaction with endothelial cells, as well as increased vascular permeability with deposits of hemosiderin; however, this model differs from the deep brain lesions demonstrated in this case series by its relationship to light exposure. ${ }^{22}$ Another plausible explanation is offered by growing evidence that ATM mediates vascular endothelial cell senescence. ${ }^{23,24}$

The imaging findings reported here in $\mathrm{A}-\mathrm{T}$ are reminiscent 

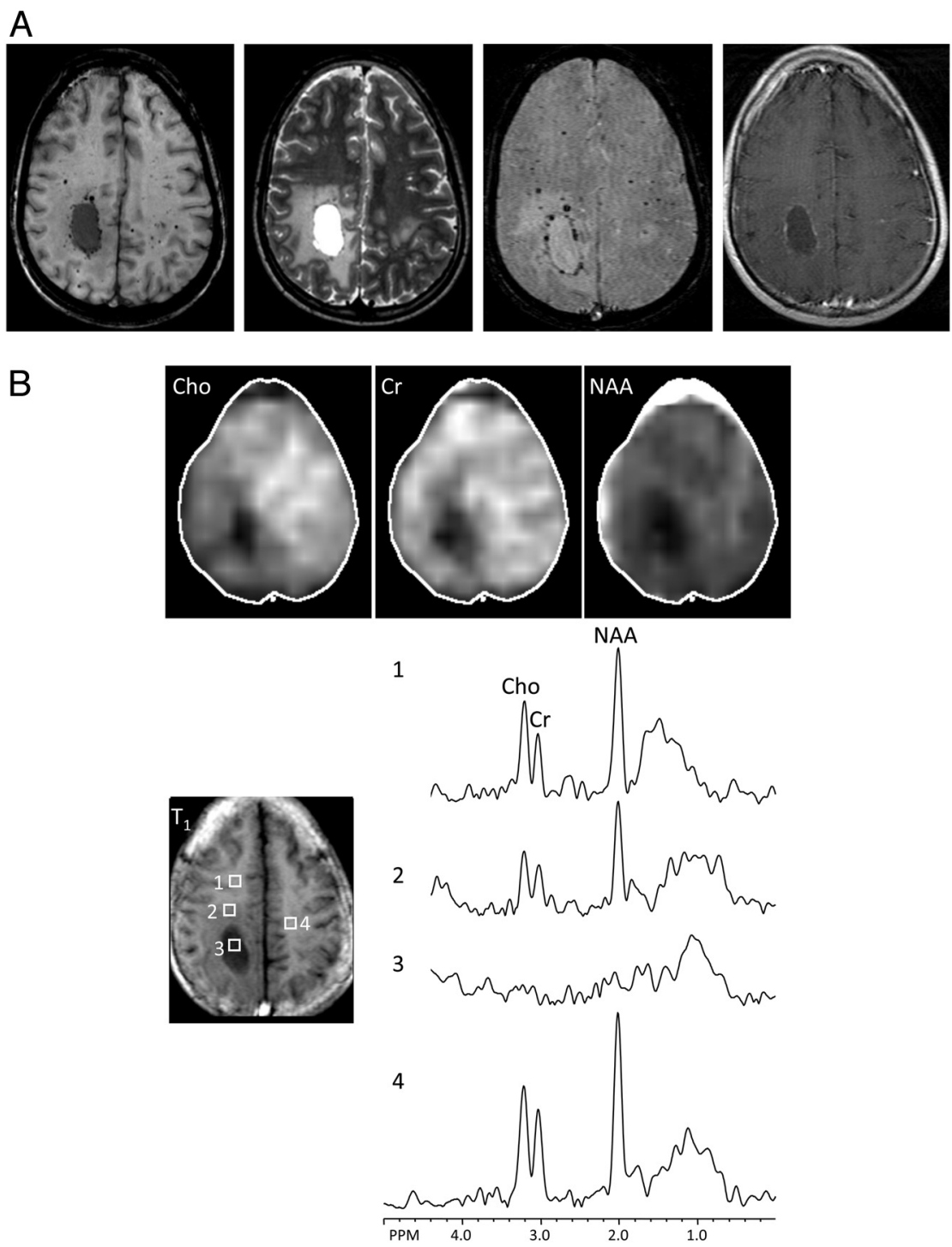

FIG 2. A 28-year-old man with no recent changes in neurologic function underwent scanning because of concern for advancing age. A, A TI hypointense, T2 hyperintense, mild rim-enhancing lesion in the right parietal white matter, with surrounding T2 hyperintensity suggestive of vasogenic edema. The contrast enhancement corresponds to punctate T2 hypointense hemosiderin deposits that outline the ovoid lesion, in addition to a few isolated foci, suggestive of telangiectasia. B, MRSI shows complete signal void in the right parietal ovoid lesion (ROI 3), compatible with a fluid collection. Within the surrounding white matter T2 hyperintensity, all metabolites are decreased (ROI 2), compared with normal-appearing white matter regions (ROIs 1 and 4). Residual lipid signals upfield from NAA are the result of head motion.

of those seen in radiation-induced vascular malformations and white matter injuries. Vascular telangiectasias are reported more frequently in patients after CNS irradiation. ${ }^{25-27}$ Parenchymal radiation-associated MR imaging changes typically manifest as progressive, mild to moderate T2 prolongation in the periventricular white matter, ${ }^{25}$ which may be the result of vascular abnormalities leading to parenchymal ischemia and white matter degeneration. It is not clear, however, whether similar mechanisms cause the white matter changes seen in older patients with A-T.

An unusual finding, identified in our 2 oldest participants (ages 34 and 28 years), was that of white matter T2/FLAIR hyperintensities. Similar hyperintensities have been the topic of individual case reports, ${ }^{11,14,15}$ although we believe that these differ from the single case report of a child with diffuse white matter signal simulating leukodystrophy, ${ }^{13}$ a pattern we have not otherwise seen. Although MR spectroscopy and MRSI have been previously reported in A-T, ${ }^{17,18}$ abnormalities (eg, reduced NAA) were found only in the atrophic cerebellum, and MR spectroscopy abnormality has not previously been reported in the supratentorial compartment. In one of the reported cases, ${ }^{14}$ these white matter signal changes were thought to represent demyelination, but the associated low level of all metabolites found in our MRSI investigation of similar T2 appearance lesions suggests that these white matter signal abnormalities represent reduced cellularity rather than active demyelination or ischemia. The peculiar appearance of a discrete fluid-filled cavity surrounded by the hemosiderin lesions in participant 10 of our series can be best explained by a transudative or exudative process related to leaky capillary telangiectasia.

A spectrum of supratentorial MR imaging abnormalities 
seem to be common in adults with A-T. These are asymptomatic, or possibly their associated signs and symptoms are lost amid the many otherwise stable neurologic impairments that such patients endure. Six of 10 participants in our current study manifested deep cerebral telangiectatic vessels best visualized on the SWI sequence, whereas the 2 oldest participants also manifested parenchymal lesions and white matter signal abnormalities. The vascular lesions have many similarities to those seen in patients after therapeutic radiation. The pathogenesis of these lesions is unknown, but candidates include impaired DNA damage response, oxidative damage, enhanced senescence, or any combination of these 3 changes. The prognosis of these MR imaging changes is unclear and will require longitudinal studies.

Disclosures: Howard Lederman—RELATED: Grant: A-T Children's Project*; Support for Travel to Meetings for the Study or Other Purposes: A-T Children's Project.* *Money paid to institution.

\section{REFERENCES}

1. Savitsky K, Bar-Shira A, Gilad S, et al. A single ataxia telangiectasia gene with a product similar to PI-3 kinase. Science 1995;268: 1749-53

2. Crawford TO. Ataxia telangiectasia. Semin Pediatr Neurol 1998;5:287-94

3. Sedgwick R, Border E. Ataxia-telangiectasia. In: de Jong J, ed. Hankbook of Clinical Neurology. Amsterdam: Elsevier 1991;347-423

4. Ciccia A, Elledge SJ. The DNA damage response: making it safe to play with knives. Mol Cell 2010;40:179-204

5. Crawford TO, Mandir AS, Lefton-Greif MA, et al. Quantitative neurologic assessment of ataxia-telangiectasia. Neurology 2000; 54:1505-09

6. Canman CE, Lim DS. The role of ATM in DNA damage responses and cancer. Oncogene 1998;17:3301-08

7. Canman CE, Lim DS, Cimprich KA, et al. Activation of the ATM kinase by ionizing radiation and phosphorylation of p53. Science 1998;281:1677-79

8. Taylor AM, Harnden DG, Arlett CF, et al. Ataxia telangiectasia: a human mutation with abnormal radiation sensitivity. Nature 1975;258:427-29

9. Cabana MD, Crawford TO, Winkelstein JA, et al. Consequences of the delayed diagnosis of ataxia-telangiectasia. Pediatrics 1998; 102:98-100
10. Farina L, Uggetti C, Ottolini A, et al. Ataxia-telangiectasia: MR and CT findings. J Comput Assist Tomogr 1994;18:724-27

11. Sardanelli F, Parodi RC, Ottonello C, et al. Cranial MRI in ataxiatelangiectasia. Neuroradiology 1995;37:77-82

12. Ciemins JJ, Horowitz AL. Abnormal white matter signal in ataxia telangiectasia. AJNR Am J Neuroradiol 2000;21:1483-85

13. Chung EO, Bodensteiner JB, Noorani PA, et al. Cerebral whitematter changes suggesting leukodystrophy in ataxia telangiectasia. J Child Neurol 1994;9:31-35

14. Habek M, Brinar VV, Rados M, et al. Brain MRI abnormalities in ataxia-telangiectasia. Neurologist 2008;14:192-95

15. Opeskin K, Waterston J, Nirenberg A, et al. Ataxia telangiectasia with long survival. J Clin Neurosci 1998;5:471-73

16. Dzieciatkowska M, Qi G, You J, et al. Proteomic characterization of cerebrospinal fluid from ataxia-telangiectasia (A-T) patients using a LC/MS-based label-free protein quantification technology. Int J Proteomics 2011;2011:578903

17. Wallis LI, Griffiths PD, Ritchie SJ, et al. Proton spectroscopy and imaging at $3 \mathrm{~T}$ in ataxia-telangiectasia. AJNR Am J Neuroradiol 2007;28:79-83

18. Lin DD, Crawford TO, Lederman HM, et al. Proton MR spectroscopic imaging in ataxia-telangiectasia. Neuropediatrics 2006;37:241-46

19. Guo Z, Kozlov S, Lavin MF, et al. ATM activation by oxidative stress. Science 2010;330:517-21

20. McKinnon PJ. ATM and ataxia telangiectasia. EMBO Rep 2004; 5:772-76

21. Raz-Prag D, Galron R, Segev-Amzaleg N, et al. A role for vascular deficiency in retinal pathology in a mouse model of ataxia-telangiectasia. Am J Pathol 2011;179:1533-41

22. Leemput J, Masson C, Bigot K, et al. ATM localization and gene expression in the adult mouse eye. Mole Vis 2009;15:393-416

23. Chen J, Goligorsky MS. Premature senescence of endothelial cells: Methusaleh's dilemma. Am J Physiol Heart Circ Physiol 2006;290:H1729-39

24. Zhan H, Suzuki T, Aizawa K, et al. Ataxia telangiectasia mutated (ATM)-mediated DNA damage response in oxidative stressinduced vascular endothelial cell senescence. $J$ Biol Chem 2010;285:29662-70

25. Gaensler EH, Dillon WP, Edwards MS, et al. Radiation-induced telangiectasia in the brain simulates cryptic vascular malformations at MR imaging. Radiology 1994;193:629-36

26. Koike S, Aida N, Hata M, et al. Asymptomatic radiation-induced telangiectasia in children after cranial irradiation: frequency, latency, and dose relation. Radiology 2004;230:93-99

27. Lew SM, Morgan JN, Psaty E, et al. Cumulative incidence of radiation-induced cavernomas in long-term survivors of medulloblastoma. J Neurosurg 2006;104:103-07 